\title{
Interferon- $\gamma$ release assays for the diagnosis of active tuberculosis: a systematic review and meta-analysis
}

\author{
M. Sester*, G. Sotgiu*, C. Lange, C. Giehl, E. Girardi, G.B. Migliori, A. Bossink, \\ K. Dheda, R. Diel, J. Dominguez, M. Lipman, J. Nemeth, P. Ravn, S. Winkler, \\ E. Huitric, A. Sandgren and D. Manissero
}

ABSTRACT: Interferon- $\gamma$ release assays (IGRAs) are now established for the immunodiagnosis of latent infection with Mycobacterium tuberculosis in many countries. However, the role of IGRAs for the diagnosis of active tuberculosis (TB) remains unclear.

Following preferred reporting items for systematic reviews and meta-analyses (PRISMA) and quality assessment of diagnostic accuracy studies (QUADAS) guidelines, we searched PubMed, EMBASE and Cochrane databases to identify studies published in January 2001-November 2009 that evaluated the evidence of using QuantiFERON-TB $\mathbb{B}_{\mathbb{B}}$ Gold in-tube (QFT-G-IT) and T-SPOT.TB directly on blood or extrasanguinous specimens for the diagnosis of active TB.

The literature search yielded 844 studies and 27 met the inclusion criteria. In blood and extrasanguinous fluids, the pooled sensitivity for the diagnosis of active TB was $80 \%(95 \% \mathrm{Cl} 75-$ $84 \%$ ) and $48 \%$ (95\% Cl 39-58\%) for QFT-G-IT, and $81 \%$ (95\% Cl 78-84\%) and $88 \%$ (confirmed and unconfirmed cases) $(95 \% \mathrm{Cl} 82-92 \%)$ for T-SPOT.TB ${ }_{\mathbb{8}}$, respectively. In blood and extrasanguinous fluids, the pooled specificity was $79 \%(95 \% \mathrm{Cl} 75-82 \%)$ and $82 \%(95 \% \mathrm{Cl} 70-91 \%)$ for QFT-G-IT, and $59 \%(95 \% \mathrm{Cl} 56-62 \%)$ and $82 \%(95 \% \mathrm{Cl} 78-86 \%)$ for T-SPOT.TB ${ }_{\mathbb{R}}$, respectively.

Although the diagnostic sensitivities of both IGRAs were higher than that of tuberculin skin tests, it was still not high enough to use as a rule out test for TB. Positive evidence for the use of IGRAs in compartments other than blood will require more independent and carefully designed prospective studies.

KEYWORDS: ECDC, interferon- $\gamma$ release assay, meta-analysis, systematic review, TBNET, tuberculosis

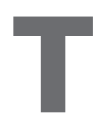
uberculosis (TB) elimination is part of the World Health Organization Stop TB Strategy [1] and the ultimate goal of the Framework Action Plan to fight TB in the European Union by The European Centre for Disease Prevention and Control (ECDC) [2]. As part of reaching the goal of $\mathrm{TB}$ elimination, individuals with active $\mathrm{TB}$ need to be rapidly identified and treated to interrupt further transmission of the infection within a population. There is a need to develop new tools for rapid case detection, evaluate their performance and provide guidance on their use for the diagnosis of $\mathrm{TB}$ to further strengthen national $\mathrm{TB}$ control programmes.

To date, rapid detection of individuals with $\mathrm{TB}$ can be difficult [3] as only $44 \%$ of all new adult cases and $15-20 \%$ of childhood cases [4] are identified by the presence of acid-fast bacilli
(AFB) in sputum smears [5]. The gold standard for the definite diagnosis of TB is the detection of Mycobacterium tuberculosis by culture, a timeconsuming method delaying the diagnosis. Moreover, diagnosis and treatment decisions may be difficult in cases with clinical suspicion of TB and negative AFB sputum smears. In this case, the rapid diagnosis of TB classically relies on the results of different methods, including the tuberculin skin test (TST), chest radiography, amplification of $M$. tuberculosis nucleic acids and/ or pathological examinations from biological specimens, although TST suffers from poor specificity in bacille Calmette-Guérin (BCG) vaccinated individuals and in those living in high TB prevalence countries.

In recent years, blood-based in vitro interferon- $\gamma$ release assays (IGRAs) have been developed as alternatives to TST for the immunodiagnosis of

\section{AFFILIATIONS}

For affiliations, please see the Acknowledgements section. ${ }^{*}$ Both authors contributed equally to this study.

CORRESPONDENCE

C. Lange

on behalf of the TBNET; Tuberculosis Center Borstel, Medical Clinic

Research Center Borstel

Parkallee 35

23845 Borstel

Germany

E-mail: clange@tz-borstel.de

Received:

July 202010

Accepted after revision:

Aug 252010

First published online:

Sept 162010 
M. tuberculosis infection. Both TST and IGRAs evaluate the presence of persistent $M$. tuberculosis-specific T-cell responses $[6,7]$ and represent indirect markers for past or present infection. The novelty of IGRAs is based on the in vitro stimulation of peripheral blood T-cells specific for the $M$. tuberculosis-specific antigens early secretory antigenic target (ESAT)-6 and culture filtrate protein (CFP)-10 [8]. The presence of reactive T-cells is assessed by the induction of interferon (IFN) $-\gamma$. Two commercialised systems are available, the QuantiFERON-TB ${ }^{\circledR}$ Gold in-tube assay (QFT-G-IT; Cellestis Ltd., Carnegie, Australia [9], also including a third antigen TB7.7) that measures IFN- $\gamma$ using an ELISA, and the TSPOT.TB $B$ assay (Oxford Immunotec Ltd., Abingdon, UK) [10] that counts cells releasing IFN- $\gamma$ with the enzyme-linked immunospot (ELISPOT) technique.

Neither IGRAs performed on blood nor TST appear to be able to distinguish between individuals with latent $M$. tuberculosis infection, active TB or past TB [11-13]. Recently, IGRAs have also been evaluated for the diagnosis of active TB directly on extrasanguinous fluids from sites of infection, including bronchoalveolar lavage fluid (BALF) [14-17], pleural effusion (PE) $[18,19]$ and cerebrospinal fluid (CSF) [20]. Up to now the accuracy for the diagnosis of TB in compartments other than blood has not been systematically evaluated.

Prompt identification and adequate treatment of new TB cases is the primary objective of $\mathrm{TB}$ control programmes. As currently available techniques are insufficient for a rapid case detection of AFB sputum smear negative cases, new diagnostic tools are urgently needed. In that respect, the impact of IGRAs applied, not only on blood, but also on extrasanguinous fluids, needs to be better evaluated before guidance on their use for the diagnosis of active TB can be developed.

To support the development of evidence-based guidance, we present the results of a systematic review and a meta-analysis on the performance of TST and commercial IGRAs from blood and, for the first time, compartments other than blood for the diagnosis of active TB.

\section{MATERIAL AND METHODS}

This systematic review was conducted according to the guidelines of the preferred reporting items for systematic reviews and meta-analyses (PRISMA) statement [21] and the quality assessment of diagnostic accuracy studies (QUADAS) checklist $[22,23]$.

\section{Search strategy}

We identified studies that evaluated the evidence of using IGRAs in order to diagnose active TB in humans. We searched PubMed, EMBASE and the Cochrane-controlled central register of controlled trials and included reports from January 2001 to November 2009. Combinations of the following search terms were applied: "tuberculosis", "T-spot", "Quantiferon", "interferon-gamma release assay", "IGRA", "ESAT-6" and "CFP-10". We restricted our search to publications in English. Unpublished sources of data were not included, as the current growing number of IGRA papers makes the presence of publication bias improbable. We also hand-searched bibliographies of retrieved articles and existing systematic reviews and meta-analyses for additional references.

\section{Study selection}

We only included studies that reported the assessment of commercially available IGRAs in individuals with a clinical suspicion of active TB, performed on blood or biological fluids other than blood (ascites, BALF, CSF, pericardial fluid, PE).

The following types of studies were excluded: 1) case reports, editorials and reviews on immunological studies; 2) laboratory studies; 3) animal studies; 4) studies performed with assays other than QFT-G-IT or T-SPOT.TB $\left.B_{\mathbb{R}} ; 5\right)$ studies not performed according to manufacturers' instructions; 6) studies in adults where pulmonary TB was not confirmed by $M$. tuberculosis culture, characteristic histopathological findings and/or nucleic acid amplification tests in $>50 \%$ of cases (in mixed studies, i.e. those without these strict criteria, data were analysed for the confirmed cases separately); 7) studies performed with cut-offs for positive test results that are not used in Europe; 8) studies where selected patients were treated for TB for $>2$ weeks prior to IGRA testing. Criteria five and seven were not applied when screening studies on IGRAs were performed on extrasanguinous fluids.

Citations were independently screened by two investigators by examining titles and abstracts to identify potentially relevant studies, and differences were resolved by consensus. These original articles were then retrieved and the full text screened for final inclusion and data extraction.

\section{Data extraction}

An electronic form for data extraction was designed. Two reviewers analysed all selected articles independently and extracted data were then crosschecked. In cases of deviations, final documentation of data was based on consenus.

The following data were extracted: calendar period of the study, country in which the study was conducted, sex distribution, enrolled age groups (adults and children), immune system impairment, BCG vaccination status, proportion of AFB sputum-positive patients, proportion of cultureconfirmed patients, the sensitivity and specificity of IGRAs on blood and specimens other than blood in the cohort or in culture-confirmed cases, proportion of indeterminate results. Although TST was not considered as a test to rule out active $\mathrm{TB}$, the sensitivity and specificity of TST was assessed in addition. If necessary, additional information not specified in the article was obtained by personal correspondence with the authors of the articles.

\section{Study quality assessment}

The inter-rater agreement obtained for the data from the included studies was $100 \%$. Finally, the quality of each study was assessed using the QUADAS (Quality Assessment of Diagnostic Accuracy Studies) checklist [22, 23]. Quality assessment of individual studies was deemed necessary to identify potential sources of bias and to limit the effects of these biases on the estimates and the conclusions of the review.

\section{Statistical analysis}

Combined estimates of sensitivity, specificity and diagnostic OR, together with their $95 \% \mathrm{CI}$, were computed collecting all the available data displayed in the selected studies (proportions of true positives, true negatives, false positives and false 
negatives). Indeterminate results were excluded before calculation of sensitivity and specificity.

Forest plots were constructed to graphically assess both the variability of the estimates of the diagnostic parameters and the weight of every sample size in the calculation of the pooled estimates (weighted means). A random-effects meta-analysis was performed in order to account for the expected betweenstudy variability for each study, along with a pooled estimate using the software for statistical analysis Stata 9.0 (StataCorp, College Station, TX, USA) and MetaDisc software, version 1.4 [24]. Inconsistency (statistical heterogeneity) among studies was assessed by the conventional Chi-squared test for heterogeneity and by calculating the $\mathrm{I}^{2}$ statistic in order to highlight the effect of true variability rather than sampling error on the overall variation in diagnostic estimates.

\section{RESULTS}

\section{Literature search}

A total of 844 studies were screened for analysis of patients with active TB using TST and/or IGRAs from blood and/or extrasanguinous fluid, of which 27 met the inclusion criteria (fig. 1) [14, 16, 17, 19, 25-47]. Of these, 9 (33.3\%) included data on IGRAs performed on extrasanguinous fluids (five BALF, three pleural fluids, one ascites) $[14,16,17,19,25,31,32,39,43]$.

\section{Characteristics of the included studies}

The studies included in the analysis were conducted in 35 different countries: South Africa was the most frequently represented country ( 6 out of 35, 17.1\%), followed by Italy and Germany (5 out of 35, 14.3\%, each), and Korea (4 out of 35, $11.4 \%$ ) (table 1 ). The majority of studies (26 out of $27,96.3 \%$ ) were carried out prospectively [14, 16, 17, 19, 25, 27-47] for an average \pm SD length of $16.8 \pm 8.7$ months; most of them (23 out of $27,85.2 \%$ ) evaluated only adults suspected of active TB [14, $16,17,19,25,27,29-37,39-44,46,47]$. The sample size varied considerably between included studies with a median (interquartile range (IQR)) of 91 (148) individuals enrolled per study. Males were more represented than females $(2,262$ versus 1,559$)$. Furthermore, 17 out of 21 (81\%) articles included known immunocompromised individuals with a median (IQR) number of 20 (38) per study (i.e. median (IQR) proportion of $28.3 \%$ $(42.1 \%))$; more than half described the use of IGRAs in HIV positive patients (14 out of $21,66.7 \%$ ) [17, 25, 30-33, 35, 40, 41, 43-47]. The mean \pm SD proportion of people immunised with BCG was $53.8 \pm 25.3 \%$, while the median (IQR) proportion of sputum smear-positive patients was 20\% (41.6\%). Age-related sub-analyses were performed (i.e. children versus adults) but characteristics, such as immunosuppression, BCG vaccination or country of origin, were often already aggregated; consequently, it was not possible to differentiate how these characteristics affect the diagnostic accuracy of IGRAs. In general, specificity data in these studies were derived from non-TB patients who were evaluated for active TB.

\section{Study quality}

Overall the quality, evaluated by the QUADAS tool, was very high and $8(66.7 \%)$ out of 12 study quality indicators were met by $100 \%$ of the included studies, thereby increasing the strength of scientific evidence of the review.

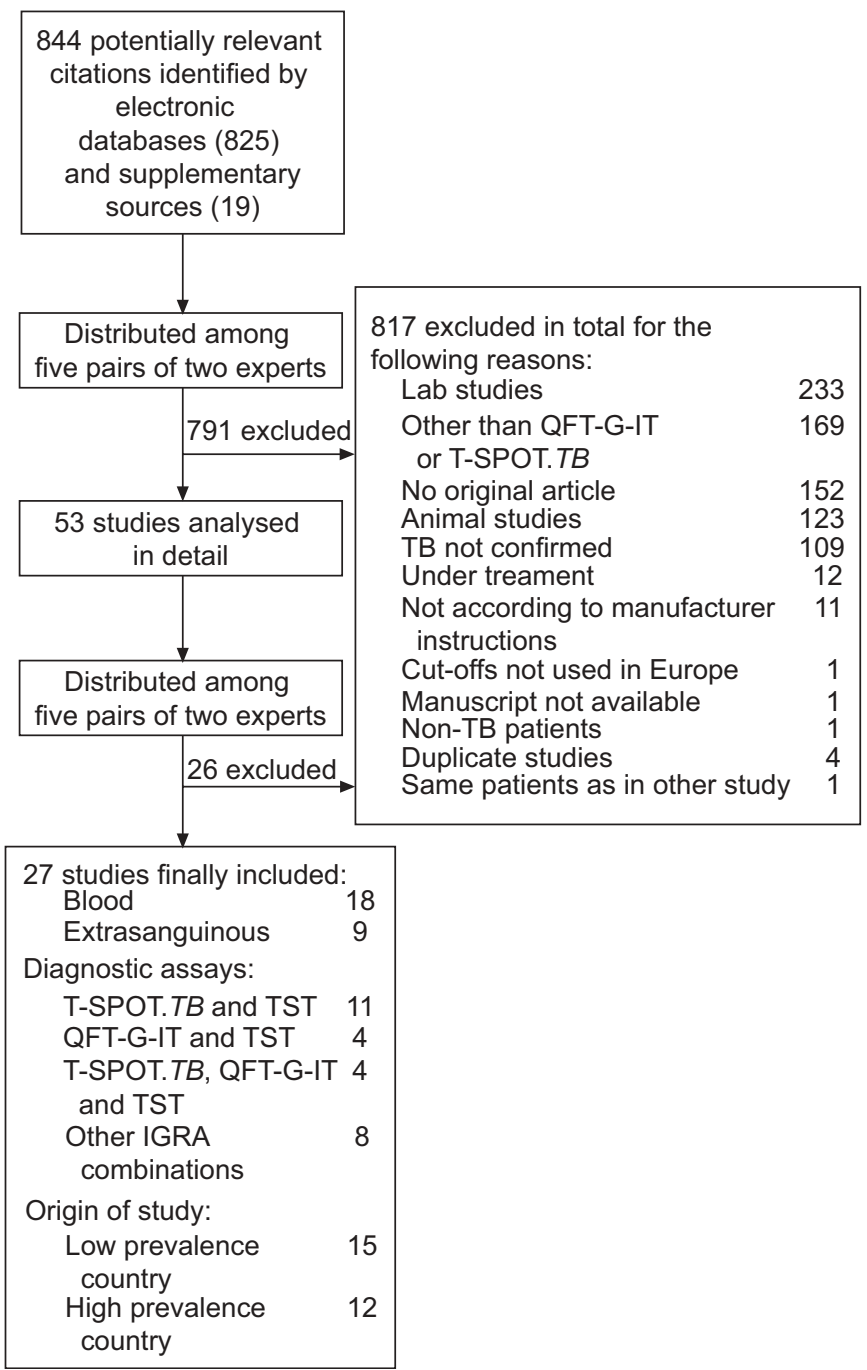

FIGURE 1. Flow diagram for study selection. Low incidence countries were defined as $<20$ in 100,000 new sputum smear-positive cases while high incidence countries were defined as $\geqslant 20$ in 100,000 new sputum smear-positive cases. QFT-G-IT: QuantiFERON-TB $\mathbb{B}$ Gold in-tube assay; TB: tuberculosis; TST: tuberculin skin test; IGRA: interferon- $\gamma$ release assay.

\section{Studies in adults}

The studies evaluating the performance of IGRAs in samples of adults were carried out in 31 different countries: mainly in South Africa and Germany (10 out of 31, 32.3\%), followed by Italy and Korea (8 out of $31,25.8 \%$ ) (table 1 ). All articles described studies based on a specific epidemiological design (23 out of $23,100 \%$ ). Males were more represented than females $(1,818$ versus 1,167). Almost half of the included subjects were immunised with BCG (mean \pm SD proportion of BCG immunised patients per study was $42.1 \pm 19.9 \%$ ). The median (IQR) proportion of sputum smear positive patients was $25 \%(45 \%)$. Patients with impaired immunity were enrolled in $15(83 \%)$ out of 18 studies, with a median (IQR) number per individual study of 24 (39.5) patients; HIV positive individuals were included in $13(86.6 \%)$ out of 15 studies. The majority of the studies enrolling adults described the performances of both TST and T-SPOT.TB ${ }_{\circledR}(10$ out of $23,43.5 \%)$. Tuberculin PPD RT-23 SSI was used by 12 (80\%) out of 15 . 
TABLE 1 Characteristics of the included studies (also stratified for adults and children)

\begin{tabular}{|c|c|c|c|}
\hline Variable & All individuals & Adults & Children \\
\hline \multicolumn{4}{|l|}{ Country ${ }^{\#}$} \\
\hline Italy & $5 / 35(14.3)$ & $4 / 31(12.9)$ & $1 / 4(25.0)$ \\
\hline Germany & $5 / 35(14.3)$ & $5 / 31(16.1)$ & \\
\hline Korea & $4 / 35(11.4)$ & $4 / 31(12.9)$ & \\
\hline Studies & $27 / 27(100.0)$ & 23/27 (85.2) & $4 / 27(14.8)$ \\
\hline Length of study months & $16.8 \pm 8.7$ & $16.2 \pm 7.9$ & $23 \pm 10.4$ \\
\hline Prospective design & 26/27 (96.3) & 23/23 (100) & $3 / 4(75)$ \\
\hline Individuals enrolled & $91(148)$ & $89(131)$ & $204.5(75.5)$ \\
\hline Studies enrolling immunocompromised patients & $17 / 21(81)$ & 15/18 (83.3) & $1 / 4(25.0)$ \\
\hline Studies enrolling HIV+ patients & $14 / 21(66.7)$ & $13 / 15(86.6)$ & $1 / 4(25.0)$ \\
\hline Proportion of BCG immunised per study & $53.8 \pm 25.3$ & $42.1 \pm 19.9$ & $74.2 \pm 21.4$ \\
\hline Number of BCG immunised per study & $62(141)$ & $27(58)$ & $201(39.75)$ \\
\hline Proportion of AFB smear positive patients & $20(41.6)$ & $25(45)$ & \\
\hline \multicolumn{4}{|l|}{ Diagnostic assays } \\
\hline T-SPOT.TB® and TST & $11 / 27(40.8)$ & 10/23 (43.5) & $1 / 4(25.0)$ \\
\hline QFT-G-IT and TST & $4 / 27(14.8)$ & $3 / 23(13.0)$ & $1 / 4(25.0)$ \\
\hline T-SPOT.TB®, QFT-G-IT and TST & $4 / 27(14.8)$ & $2 / 23(8.7)$ & $2 / 4(50.0)$ \\
\hline Others (IGRAs only) & $8 / 27(29.6)$ & $8 / 23(34.8)$ & \\
\hline
\end{tabular}

Data are presented as $\mathrm{n} / \mathrm{n}$ total (\%), mean \pm SD or median (interquartile range), unless otherwise stated. BCG: bacille Calmette-Guérin; AFB: acid fast bacilli; TST: tuberculin skin test; QFT-G-IT: QuantiFERON-TB® Gold in-tube; IGRA: interferon- $\gamma$ release assay. ${ }^{\#}$ : 35 countries contributed to 27 studies.

\section{Studies in children}

Four articles included paediatric patients [26, 28, 38, 45] of which $2(50 \%)$ were derived from the UK (table 1$)$. More than half ( 3 out of $4,75 \%$ ) planned a prospective analysis. The median (IQR) number of children enrolled per study was 204.5 (75.5), with a male:female ratio of 444:392. Most of them were vaccinated with BCG (mean proportion of BCG-immunised individuals per study: $74.2 \%(21.4 \%)$ ). Only $1(25 \%)$ out of 4 studies enrolled immunocompromised children. $50 \%$ ( 2 out of 4) of the selected studies evaluated the performances of TSPOT.TB $B$, QFT-G-IT and TST at the same time. Tuberculin PPD RT-23 SSI was used by 3 out of 4 studies $(75 \%)$ and reaction size was read after $48-72 \mathrm{~h}$ (4 out of $4,100 \%$ ).

\begin{tabular}{|c|c|c|}
\hline TABLE 2 & \multicolumn{2}{|c|}{$\begin{array}{l}\text { Characteristics of the tuberculin skin test (TST) } \\
\text { described in the included studies }\end{array}$} \\
\hline \multicolumn{3}{|l|}{ TST used } \\
\hline 2 IU RT23 & & $12 / 19(63.1)$ \\
\hline \multicolumn{2}{|c|}{ TST reading after $48-72 \mathrm{~h}$} & 8/18 (44.4) \\
\hline \multicolumn{2}{|c|}{ Individuals who underwent TST } & $98(131)$ \\
\hline \multicolumn{2}{|c|}{ Culture-confirmed cases who underwent TST n } & 473 \\
\hline \multicolumn{2}{|c|}{ Culture-confirmed cases who underwent TST per study } & $31(24.5)$ \\
\hline
\end{tabular}

Data are presented as $n / n$ total (\%) or median (interquartile range), unless otherwise stated.

\section{Characteristics of the TST described in the included studies}

The median (IQR) number of individuals who underwent TST was 98 (131) (table 2). The reaction was read at $48-72 \mathrm{~h}$ in almost half of the cases (44.4\%). Pooled sensitivity of the TST was $65 \%\left(95 \%\right.$ CI $61-68 \%$; $\left.\mathrm{I}^{2}=89 \%\right)$ for all patients with TB (confirmed and non-confirmed by culture), while pooled specificity was $75 \%$ (95\% CI $72-78 \%$; $\mathrm{I}^{2}=89 \%$ ) (fig. 2). Pooled diagnostic OR was 5.72 (95\% CI 3.78-8.65; $\mathrm{I}^{2}=46.1 \%$ ).

Pooled sensitivity computed after the inclusion of the studies not showing specificity $(n=4)$ was similar to the aforementioned figure (i.e., $66 \%$; $95 \%$ CI $63-69 \% ; \mathrm{I}^{2}=93.2 \%$ ), while pooled specificity computed after the inclusion of the studies not showing sensitivity $(\mathrm{n}=2)$ was lower at $66 \%(95 \%$ CI $63-$ $\left.68 \% ; \mathrm{I}^{2}=94.3 \%\right)$. Pooled sensitivity was $68 \%(95 \%$ CI $63-72 \%$; $\mathrm{I}^{2}=90 \%$ ) when only patients with culture-confirmed $\mathrm{TB}$ were evaluated.

\section{Characteristics of IGRAs performed on blood samples}

The median (IQR) number of individuals who underwent QFT-G-IT was 94.5 (189). The total number of TB patients and controls with determinate results were 1,035 and 569, respectively. The pooled sensitivity was $80 \%$ (95\% CI $75-$ $84 \% ; \mathrm{I}^{2}=45.3 \%$ ) for all patients with TB (confirmed and nonconfirmed by culture) (fig. 3). Pooled specificity was $79 \%(95 \%$ CI $\left.75-82 \% ; \mathrm{I}^{2}=81.1 \%\right)$. Pooled diagnostic OR was $11.5(95 \% \mathrm{CI}$ 5.1-25.7; $\left.\mathrm{I}^{2}=67.8 \%\right)$. The addition of the studies not showing specificity $(n=5)$ did not dramatically change the pooled 
a)

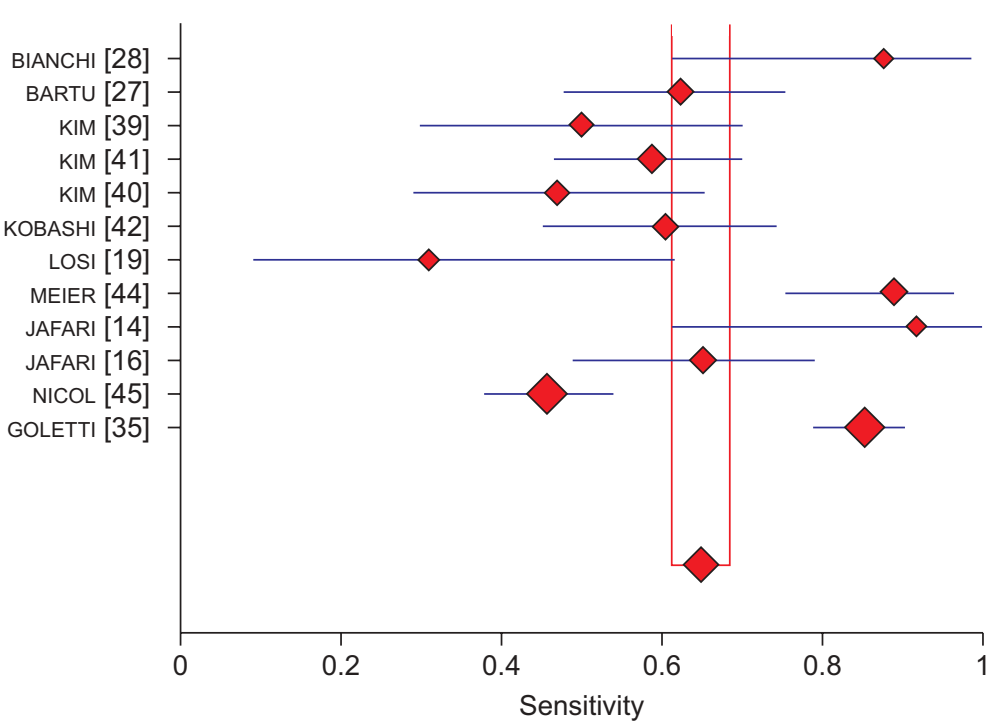

b)

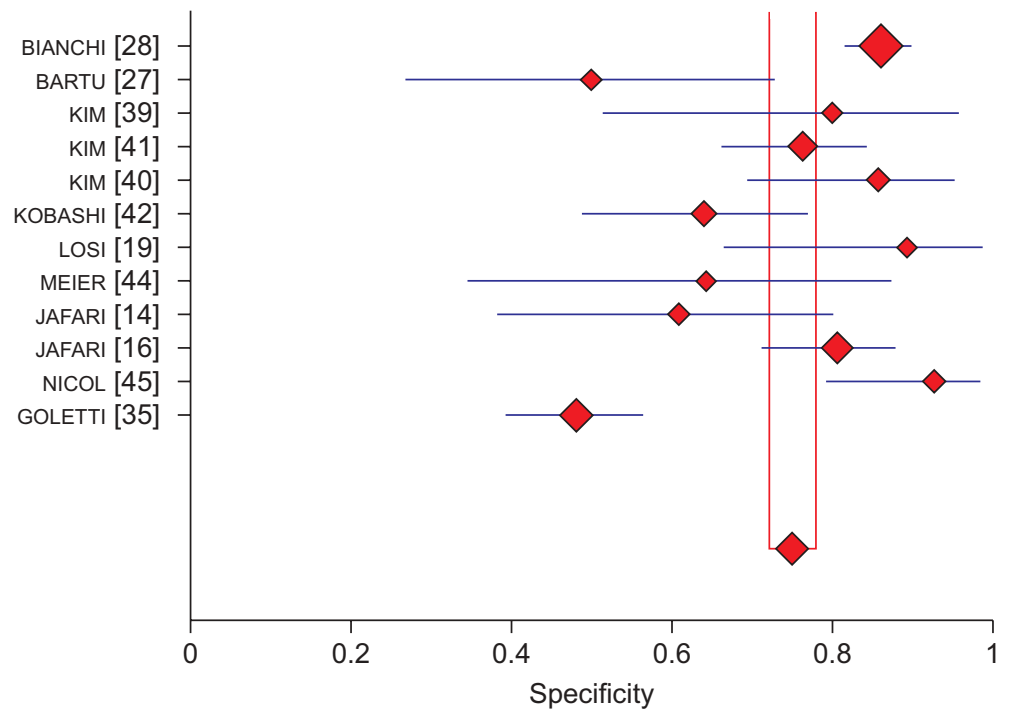

c)

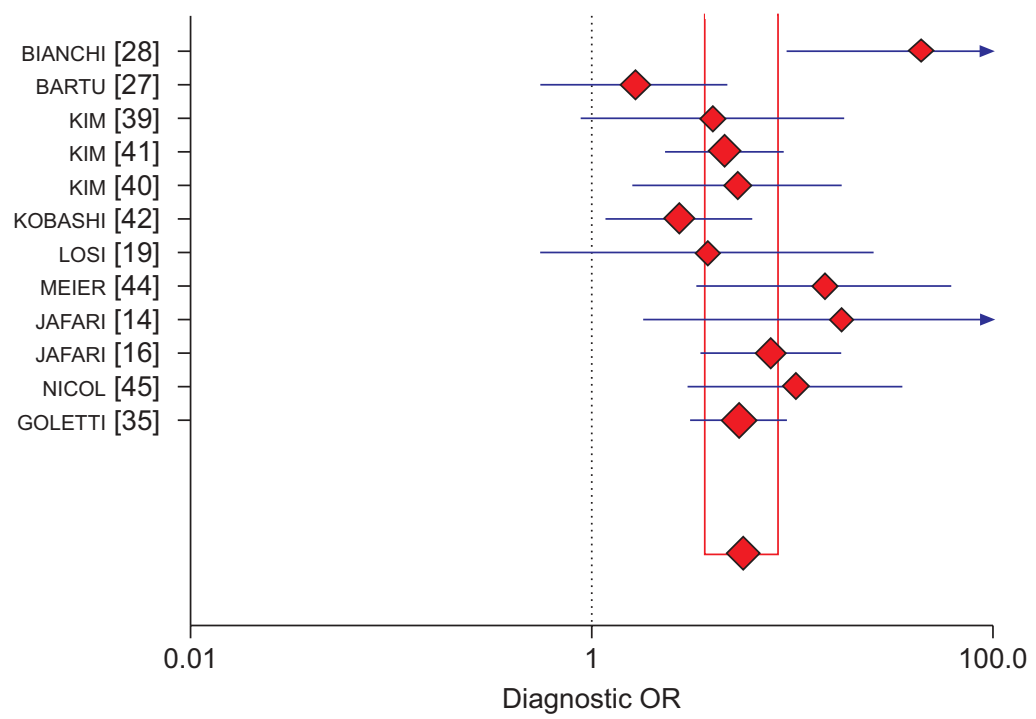

Sensitivity

$95 \% \mathrm{Cl}$

0.88

0.62

0.62-0.98

$0.48-0.75$

0.30-0.70

0.50

0.47

$0.47-0.70$

$0.29-0.65$

0.60

$0.45-0.74$

$0.09-0.61$

0.76-0.96

$0.62-1.00$

$0.49-0.79$

$0.38-0.54$

0.79-0.90

0.85
Patients $\mathrm{n} / \mathrm{n}$

$14 / 16$

$33 / 52$

$13 / 26$

$44 / 75$

$15 / 32$

29/48

$4 / 13$

$40 / 45$

$11 / 12$

$28 / 43$

$75 / 164$

$151 / 177$
Pooled sensitivity $=0.65(0.61-0.68)$ Chi-squared $=99.79 ; d f=11 \quad(p=0.0000)$ Inconsistency $\mathrm{I}^{2}=89.0 \%$

$\begin{array}{ccc}\text { Specificity } & 95 \% \mathrm{Cl} & \text { Patients } \mathrm{n} / \mathrm{n} \\ 0.86 & 0.82-0.90 & 276 / 320 \\ 0.50 & 0.27 \_0.73 & 10 / 20 \\ 0.80 & 0.52-0.96 & 12 / 15 \\ 0.76 & 0.67-0.84 & 74 / 97 \\ 0.86 & 0.70-0.95 & 30 / 35 \\ 0.64 & 0.49-0.77 & 32 / 50 \\ 0.89 & 0.67-0.99 & 17 / 19 \\ 0.64 & 0.35-0.87 & 9 / 14 \\ 0.61 & 0.39-0.80 & 14 / 23 \\ 0.81 & 0.72-0.88 & 83 / 103 \\ 0.93 & 0.80-0.98 & 37 / 40 \\ 0.48 & 0.40-0.56 & 72 / 150\end{array}$

Pooled specificity $=0.75(0.72-0.78)$ Chi-squared $=101.61 ; d f=11 \quad(p=0.0000)$ Inconsistency $\left.\right|^{2}=89.2 \%$

$\begin{array}{cc}\text { Diagnostic OR } & 95 \% \mathrm{Cl} \\ 43.91 & 9.65-199.84 \\ 1.65 & 0.58-4.66 \\ 4.00 & 0.91-17.58 \\ 4.57 & 2.37-8.80 \\ 5.29 & 1.64-17.13 \\ 2.71 & 1.20-6.14 \\ 3.78 & 0.58-24.75 \\ 14.40 & 3.43-60.48 \\ 17.11 & 1.87-156.26 \\ 7.75 & 3.50-17.15 \\ 10.39 & 3.08-35.06 \\ 5.36 & 3.17-9.06\end{array}$

Pooled diagnostic OR=5.72 (3.78-8.65) Cochran- $Q=20.42 ; d f=11 \quad(p=0.0399)$ Inconsistency $\mathrm{I}^{2}=46.1 \%$ Tau-squared $=0.2177$

FIGURE 2. Figure legend presented on following page. 


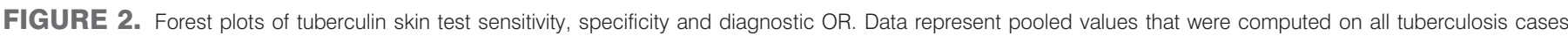

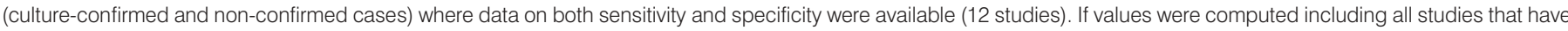

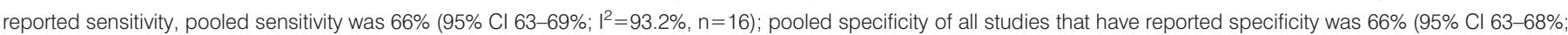
$\left.1^{2}=94.3 \%, n=14\right)$. df: degrees of freedom.

sensitivity (i.e., 77\%; 95\% CI 75-80\%; $\mathrm{I}^{2}=64.5 \%$ ). Pooled sensitivity was $81 \%\left(95 \%\right.$ CI $78-84 \%$; $\left.\mathrm{I}^{2}=0 \%\right)$ for all patients with culture-confirmed TB.

The median (IQR) proportion of QFT-G-IT indeterminate results was $7 \%(12.6 \%)$. This was two-times lower than in the samples derived from adults only $(14.9 \%(13.6 \%))$. The median (IQR) proportion of indeterminate results among children was $6.3 \%(3.6 \%)$. The number of children with determinate results was 491 . Mean \pm SD sensitivity in children was $79.9 \pm 20.9 \%$; specificity was reported in only one paediatric study $(85.8 \%)$.

The median (IQR) number of individuals who underwent TSPOT.TB ${ }_{\mathbb{B}}$ was 90 (138). The total number of TB patients and controls with determinate results were 1,212 and 1,070, respectively. Pooled T-SPOT.TB ${ }_{\circledR}$ sensitivity was $81 \%(95 \%$ CI $78-84 \%$; $\mathrm{I}^{2}=93.3 \%$ ) for all patients with TB (confirmed and non-confirmed by culture) (fig. 4). The inclusion of the papers not showing specificity $(n=4)$ did not modify the aforementioned pooled T-SPOT.TB ${ }^{\circledR}$ sensitivity (i.e., 83\%, 95\% CI $80-85 \%$; I $\left.{ }^{2}=94.2 \%\right)$. Pooled T-SPOT.TB ${ }$ sensitivity was $92 \%$ (95\% CI 90-93\%; I I $=78 \%$ ) when patients with culture-confirmed TB were evaluated independently. Pooled T-SPOT.TB specificity was $59 \%\left(95 \%\right.$ CI $\left.56-62 \% ; \mathrm{I}^{2}=84.5 \%\right)$ while the pooled diagnostic OR was higher than that of QFT-G-IT at 18.86 (95\% CI 8.7-40.7; $\left.\mathrm{I}^{2}=81.2 \%\right)$.

The median (IQR) proportion of T-SPOT.TB $B_{\mathbb{R}}$ indeterminate results was $3.4 \%(5 \%)(5 \%(9.5 \%)$ in adults and $8 \%(2.5 \%)$ in children). A total of 227 children with $\mathrm{TB}$ and valid $\mathrm{T}$ SPOT.TB $B_{\circledast}$ results were evaluated. Mean \pm SD sensitivity was $42.2 \pm 11 \%$ in all cases. Specificity was computed in only one study $(74 \%)$.

\section{Characteristics of IGRAs performed on extrasanguinous samples}

Nine articles described the diagnostic performance of IGRAs on extrasanguinous fluids: five on pleural fluid $[19,25,31,32$, 43], three on BALF [14, 16, 17], and one on ascitic fluid [39]. None of these studies included children.

The total number of culture-confirmed cases undergoing QFT-G-IT was 64 with a median (IQR) number of 18 (24) per single study. Pooled sensitivity and specificity for all TB cases (culture-confirmed and non-confirmed) were 48\% (95\% CI 39$58 \% ; \mathrm{I}^{2}=$ not assessed) and $82 \%\left(95 \%\right.$ CI $70-91 \% ; \mathrm{I}^{2}=$ not assessed), respectively (fig. 5). Pooled sensitivity for cultureconfirmed TB cases was 52\% (95\% CI 39-64\%; $\mathrm{I}^{2}=38 \%$ ). Pooled diagnostic OR was 3.84 (95\% CI 1.73-8.5; $\mathrm{I}^{2}=$ not assessed). The proportion of QFT-G-IT indeterminate results was very high, with a median (IQR) of $23.1 \%(40.1 \%)$.

The total number of culture-confirmed cases undergoing TSPOT.TB ${ }$ was 128 with a median (IQR) number of 17 (26) in each study. Pooled sensitivity of the T-SPOT.TB ${ }_{\circledR}$ for all TB cases (culture-confirmed and non-confirmed) was 88\% (95\% CI $82-92 \%$; $\mathrm{I}^{2}=57.9 \%$ ), comparable to the pooled sensitivity for culture-confirmed TB cases of $88 \%$ (95\% CI $81-93 \%$; $\mathrm{I}^{2}=22 \%$ ).
Pooled specificity was $82 \%$ (95\% CI 78-86\%; $\mathrm{I}^{2}=71.5 \%$ ) (fig. 6). Pooled diagnostic OR, higher than that computed for QFT-GIT, was 35.83 (95\% CI 15.6-82.4; $\mathrm{I}^{2}=30.8 \%$ ). The median (IQR) proportion of T-SPOT.TB ${ }_{\mathbb{R}}$ indeterminate results was lower $(5 \%(9.8 \%))$ than that of the QFT-G-IT group.

\section{DISCUSSION}

Evidence for the use of the IGRAs (T-SPOT.TB $B_{\circledR}$ and QFT-G-IT) and TST for diagnosing active TB was assessed by a systematic review and meta-analysis of published literature. It is an updated analysis of studies that have used IGRAs on whole blood [3, 48, 49], and brings added value by including, for the first time, an evaluation of IGRA performance in extrasanguinous fluids. Three important messages may be derived from this analysis. First, although the diagnostic sensitivity of both IGRAs was higher than that of TST for the diagnosis of active TB, it was still not high enough to use these assays as a rule out test for TB. Secondly, the specificity of IGRAs for the diagnosis of TB was much lower than previously reported $[3,48-50]$ and inadequate to distinguish active TB from latent infection. Thirdly, the data indicate that the application of the T-SPOT.TB ${ }_{\circledR}$ assay to extrasanguinous fluids is currently the best available immunodiagnostic method for the diagnosis of active TB, although the studies were few and number of study subjects limited.

In line with previous analyses, the sensitivity of both IGRAs for the diagnosis of active TB was higher than that of TST. Overall sensitivities did not differ between T-SPOT.TB $B_{\mathbb{R}}$ and QFT-G-IT. In general, however, the diagnostic sensitivity of IGRAs is too low to support their use as a rule-out test for TB, as the negative predictive value would not be sufficiently high. This low diagnostic sensitivity may, in part, be a consequence of a significant heterogeneity between study results (i.e. due to advanced TB, co-morbidity, high versus low burden setting). A combination of one of the two IGRAs with the TST or other investigations such as smear microscopy or chest radiography, may increase the post-test probability of ruling out active TB [35, $38,51]$, but further studies are needed to evaluate this approach.

Interestingly, the pooled specificities of T-SPOT.TB ${ }_{\Re}$, QFT-G-IT and TST in this analysis were as low as 59\%,79\% and $75 \%$, respectively. Unlike previous reports on the specificity of IGRAs for the diagnosis of TB that have exclusively used control groups with a low risk of M. tuberculosis infection [48], we have now also included studies that have assessed $\mathrm{T}$ SPOT.TB ${ }$, QFT-G-IT and TST in suspects of active TB who were found to have an alternative disease. Apparently, the low specificity is due to the fact that many of these individuals have positive IGRA or TST results compatible with a diagnosis of latent M. tuberculosis infection. Unlike low-risk controls, these are more representative of patients that would be tested in a routine clinical setting. Thus, the low specificity obtained in this analysis emphasises that no current immunodiagnostic test is suitable to distinguish active TB from latent infection with $M$. tuberculosis in clinical practice when using peripheral blood. This is an important finding that should be acknowledged 
a)

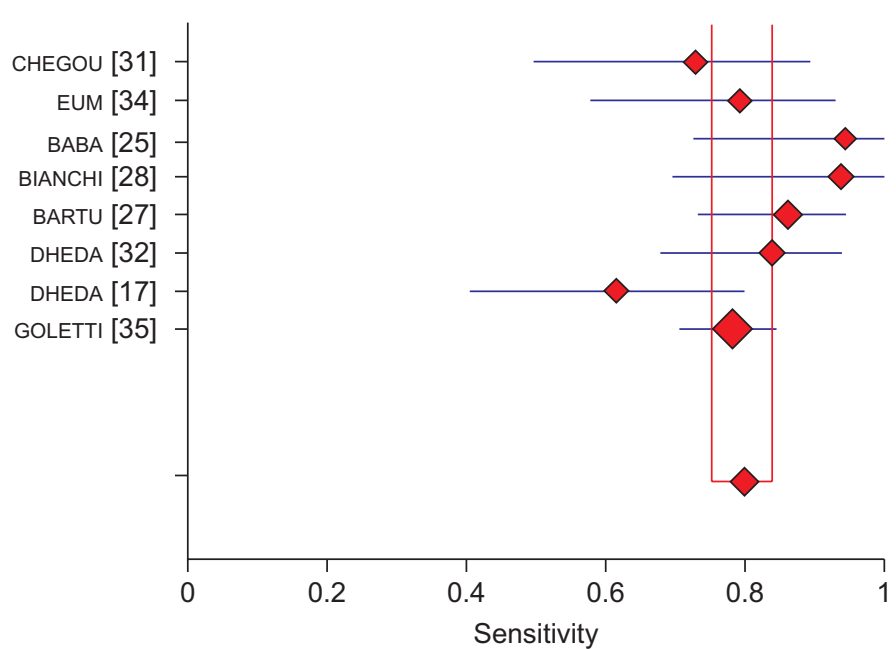

$\begin{array}{ccc}\text { Sensitivity } & 95 \% \mathrm{Cl} & \text { Patients } \mathrm{n} / \mathrm{n} \\ 0.73 & 0.50-0.89 & 16 / 22 \\ 0.79 & 0.58-0.93 & 19 / 24 \\ 0.94 & 0.73-1.00 & 17 / 18 \\ 0.94 & 0.70-1.00 & 15 / 16 \\ 0.86 & 0.73-0.94 & 43 / 50 \\ 0.84 & 0.68-0.94 & 31 / 37 \\ 0.62 & 0.41-0.80 & 16 / 26 \\ 0.78 & 0.71-0.84 & 121 / 155\end{array}$

Pooled sensitivity $=0.80(0.75-0.84)$

Chi-squared $=12.80 ; d f=7 \quad(p=0.0770)$ Inconsistency $\mathrm{I}^{2}=45.3 \%$

b)

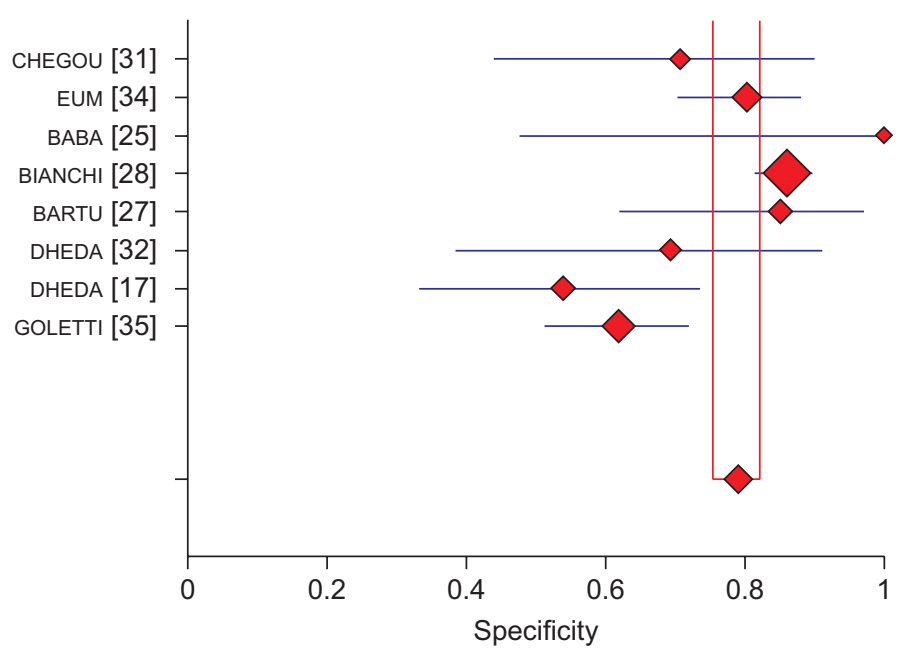

$\begin{array}{ccc}\text { Specificity } & 95 \% \mathrm{Cl} & \text { Patients } \mathrm{n} / \mathrm{n} \\ 0.71 & 0.44-0.90 & 12 / 17 \\ 0.80 & 0.71-0.88 & 55 / 73 \\ 1.00 & 0.48-1.00 & 5 / 5 \\ 0.86 & 0.82-0.89 & 273 / 318 \\ 0.85 & 0.62-0.97 & 17 / 20 \\ 0.69 & 0.39-0.91 & 9 / 13 \\ 0.54 & 0.33-0.73 & 14 / 26 \\ 0.62 & 0.51-0.72 & 60 / 97\end{array}$

Pooled specificity $=0.79(0.75-0.82)$

Chi-squared $=37.10 ; d f=7 \quad(p=0.0000)$ Inconsistency $\mathrm{I}^{2}=81.1 \%$

c)

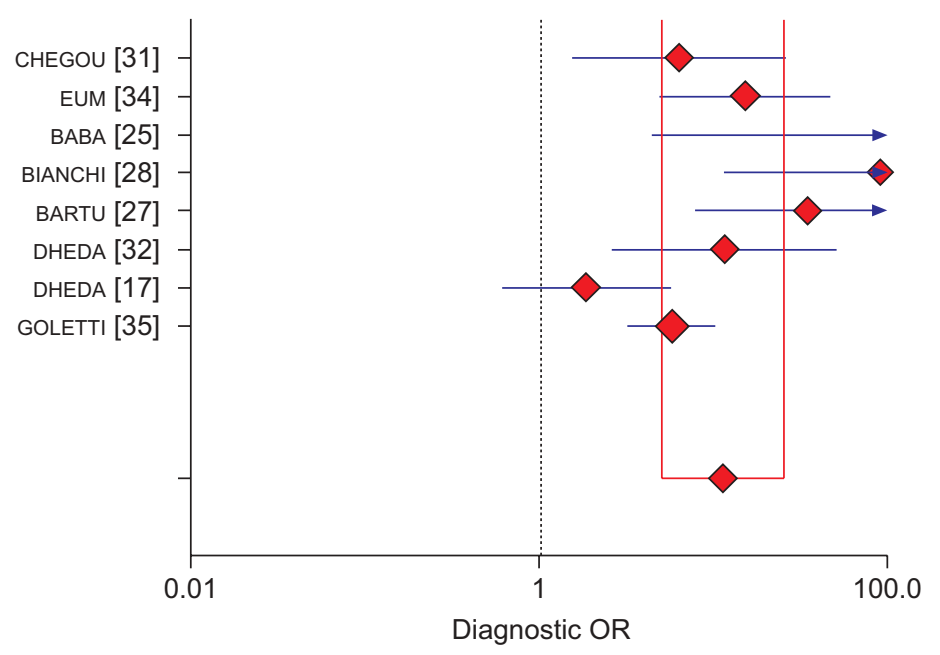

$\begin{array}{cc}\text { Diagnostic OR } & 95 \% \mathrm{Cl} \\ 6.40 & 1.57-26.03 \\ 15.41 & 5.07-46.86 \\ 128.33 & 4.54-3624.88 \\ 91.00 & 11.73-705.93 \\ 34.81 & 8.05-150.56 \\ 11.63 & 2.68-50.40 \\ 1.87 & 0.62-5.63 \\ 5.77 & 3.30-10.09\end{array}$

Pooled diagnostic $\mathrm{OR}=11.47(5.12-25.69)$

Cochran- $\mathrm{Q}=21.71 ; \mathrm{df}=7 \quad \mathrm{p}=0.0028)$

Inconsistency $\mathrm{I}^{2}=67.8 \%$

Tau-squared $=0.8101$

FIGURE 3. Forest plots of sensitivity, specificity and diagnostic odds ratio of QuantiFERON-TB® Gold in-tube performed on blood samples. Data represent pooled values that were computed on all tuberculosis cases (culture-confirmed and non-confirmed cases) where data on both sensitivity and specificity were available (8 studies). If values were computed including all studies that have reported sensitivity ( 13 studies), pooled sensitivity was $77 \%\left(95 \% \mathrm{Cl} 75-80 \% ; \mathrm{I}^{2}=64.5 \%\right)$. df: degrees of freedom. 
a)

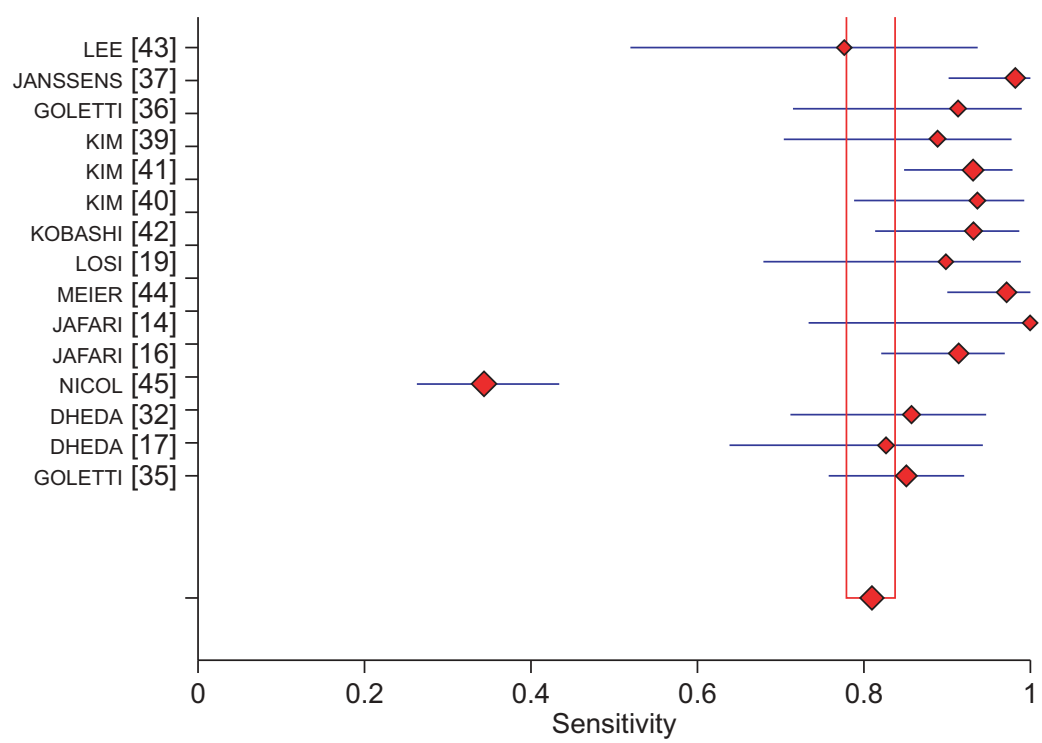

b)

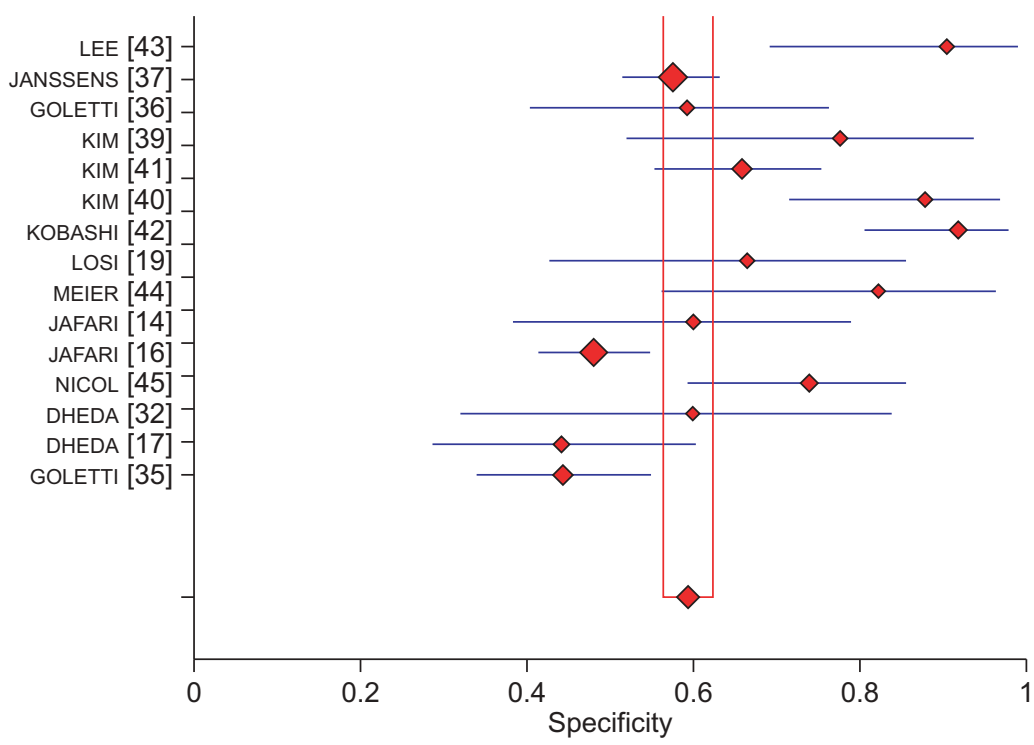

c)

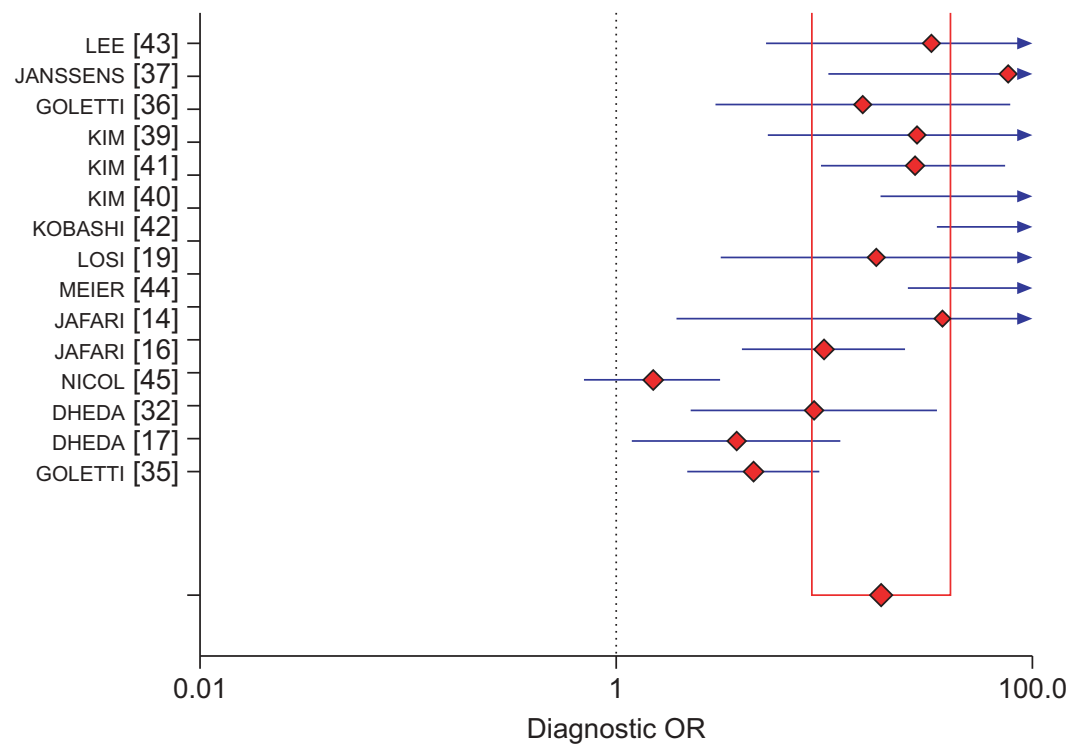

Sensitivity

$95 \% \mathrm{Cl}$

0.78

0.52-0.94

$0.91-1.00$

0.72-0.99

$0.71-0.98$

$0.85-0.98$

$0.79-0.99$

$0.82-0.99$

0.68-0.99

$0.90-1.00$

0.74-1.00

$0.83-0.97$

$0.27-0.43$

$0.71-0.95$

$0.64-0.94$

$0.76-0.92$

Patients $n / n$

$14 / 18$

$57 / 58$

$24 / 27$

$24 / 27$

$70 / 75$

$30 / 32$

$42 / 45$

$18 / 20$

$70 / 72$

$12 / 12$

$65 / 71$

$46 / 133$

$36 / 42$

$24 / 29$

$75 / 88$

Pooled sensitivity $=0.81(0.78-0.84)$

Chi-squared $=208.78 ; d f=14(p=0.0000)$

Inconsistency $\left.\right|^{2}=93.3 \%$

$\begin{array}{cc}\text { Specificity } & 95 \% \mathrm{Cl} \\ 0.90 & 0.70-0.99 \\ 0.58 & 0.52-0.63 \\ 0.59 & 0.41-0.76 \\ 0.78 & 0.52-0.94 \\ 0.66 & 0.56-0.75 \\ 0.88 & 0.72-0.97 \\ 0.92 & 0.81-0.98 \\ 0.67 & 0.43-0.85 \\ 0.82 & 0.57-0.96 \\ 0.60 & 0.39-0.79 \\ 0.48 & 0.42-0.55 \\ 0.74 & 0.60-0.85 \\ 0.60 & 0.32-0.84 \\ 0.44 & 0.29-0.60 \\ 0.44 & 0.34-0.55\end{array}$

Patients $\mathrm{n} / \mathrm{n}$

$19 / 21$

$176 / 306$

$19 / 32$

$14 / 18$

$64 / 97$

$29 / 33$

$46 / 50$

$14 / 21$

$14 / 17$

$15 / 25$

$118 / 245$

$37 / 50$

$9 / 15$

$19 / 43$

$43 / 97$

Pooled specificity $=0.59(0.56-0.62)$

Chi-squared $=90.27 ; d f=14(p=0.0000)$ Inconsistency $\mathrm{I}^{2}=84.5 \%$

Diagnostic OR

33.25
77.17
15.35
28.00
27.15
108.75
161.00
18.00
163.33
36.90
10.07
1.50
9.00
3.80
4.59

33.25

15.35

28.00

161.00

36.90

10.07

3.80
4.59

Pooled diagnostic OR=18.86 (8.72-40.77)

Cochran- $Q=74.44 ; d f=14 \quad(p=0.0000)$

Inconsistency $\mathrm{I}^{2}=81.2 \%$

Tau-squared $=1.7259$

FIGURE 4. Figure legend presented on following page. 
FIGURE 4. Forest plots of sensitivity, specificity and diagnostic odds ratio of T-SPOT.TB® performed on blood samples. Data represent pooled values that were computed on all tuberculosis cases (culture-confirmed and non-confirmed cases) where data on both sensitivity and specificity were available (15 studies). If values were computed including all studies that have reported sensitivity ( 19 studies), pooled sensitivity was $83 \%\left(95 \% \mathrm{Cl} 80-85 \%\right.$; $\left.I^{2}=94.2 \%\right)$. Note that the pooled sensitivity of TSPOT.TB $₫$ was obtained including the sensitivity derived from the study by Nicol et al. [45], which is significantly lower $(<40 \%)$ than that described in the other studies. df: degrees of freedom.

in future recommendations on the use of IGRAs for the diagnosis of M. tuberculosis infection.

The situation is different in extrasanguinous samples, where the pooled sensitivity was significantly higher for T-SPOT.TB (88\%) compared with QFT-G-IT (48\%). In addition, the specificity of T-SPOT.TB $B_{\circledR}$ in extrasanguinous samples was higher compared to that recorded in blood ( $82 \%$ versus $59 \%$ ), whereas specificity of QFT-G-IT did not differ in the two compartments (79\% versus $82 \%)$. The median proportion of indeterminate results with T-SPOT.TB® was similar in blood and extrasanguinous fluids, whereas QFT-G-IT had more indeterminate results in extrasanguinous fluids. Thus, both the higher sensitivity and the lower proportion of indeterminate results indicate superiority of the T-SPOT.TB $B_{\circledR}$ when analysing extrasanguinous samples from patients with active TB. a)

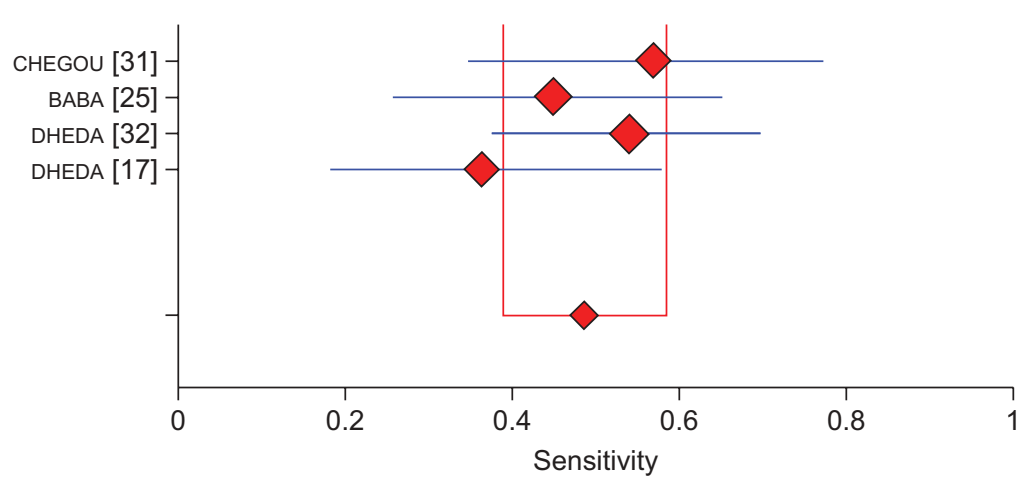

b)

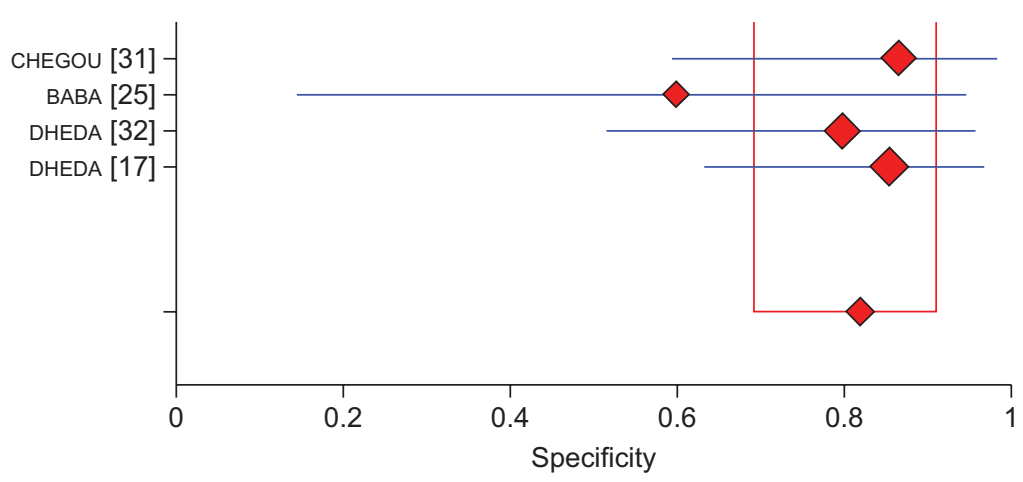

c)

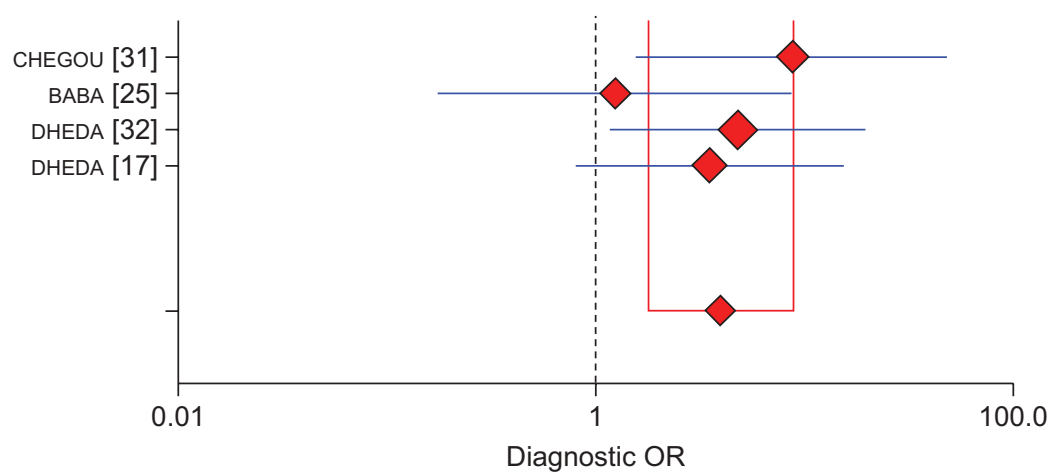

$\begin{array}{ccc}\text { Sensitivity } & 95 \% \mathrm{Cl} & \text { Patients } \mathrm{n} / \mathrm{n} \\ 0.57 & 0.34-0.77 & 13 / 23 \\ 0.44 & 0.25-0.65 & 12 / 27 \\ 0.54 & 0.37-0.69 & 22 / 41 \\ 0.36 & 0.18-0.57 & 9 / 25\end{array}$

Pooled sensitivity $=0.48(0.39-0.58)$

Chi-squared $=2.79 ; \mathrm{df}=3(\mathrm{p}=0.4244)$ Inconsistency $\mathrm{I}^{2}=0.0 \%$

$\begin{array}{ccc}\text { Specificity } & 95 \% \mathrm{Cl} & \text { Patients } \mathrm{n} / \mathrm{n} \\ 0.87 & 0.60-0.98 & 13 / 15 \\ 0.60 & 0.15-0.95 & 2 / 5 \\ 0.80 & 0.52-0.96 & 12 / 15 \\ 0.86 & 0.64-0.97 & 18 / 21\end{array}$

Pooled specificity $=0.82(0.70-0.91)$

Chi-squared $=1.81 ; d f=3(p=0.6138)$ Inconsistency $\mathrm{I}^{2}=0.0 \%$

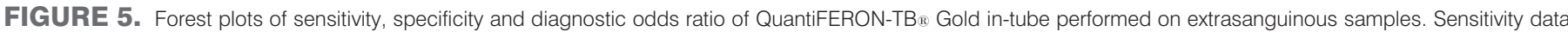

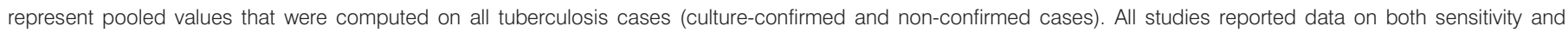
specificity. df: degrees of freedom. 
a)

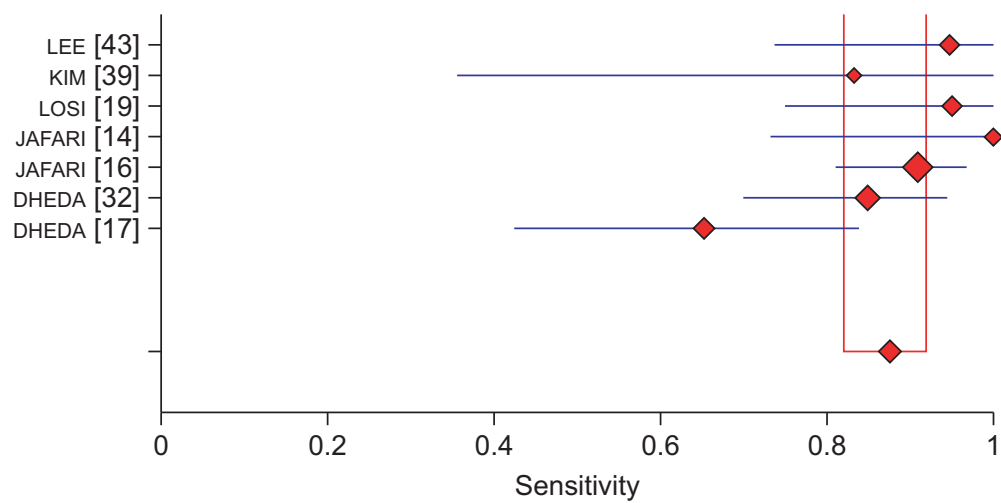

b)

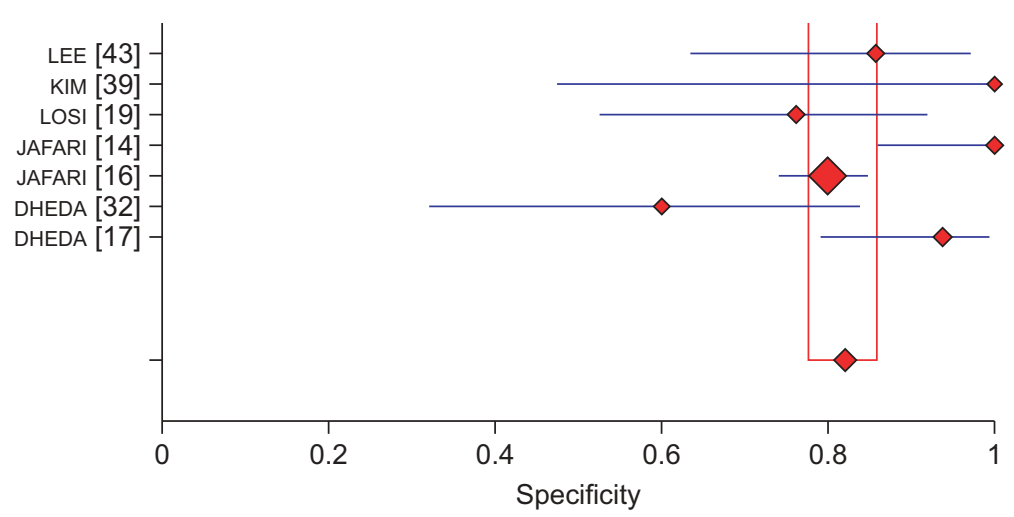

Sensitivity
0.95
0.83
0.95
1.00
0.91
0.85
0.65

$95 \% \mathrm{Cl}$

$0.74-1.00$

$0.36-1.00$

$0.75-1.00$

$0.74-1.00$

$0.81-0.97$

$0.70-0.94$

$0.43-0.84$

Pooled sensitivity $=0.88(0.82-0.92)$

Chi-squared $=14.25 ; \mathrm{df}=6 \quad(p=0.0270)$ Inconsistency | ${ }^{2}=57.9 \%$

\section{Patients $\mathrm{n} / \mathrm{n}$ \\ $18 / 19$ \\ $5 / 6$ \\ $19 / 20$ \\ $12 / 12$ \\ $60 / 66$ \\ $34 / 40$ \\ $15 / 23$}

c)

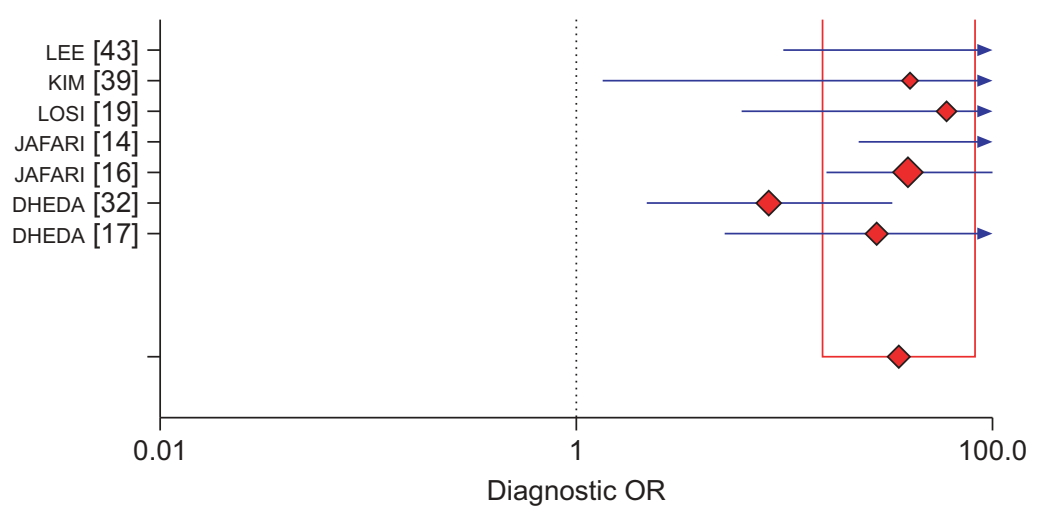

Patients $\mathrm{n} / \mathrm{n}$ $18 / 21$

$5 / 5$

$16 / 21$

$25 / 25$

$199 / 249$

$9 / 15$

$30 / 32$

Pooled specificity $=0.82(0.78-0.86)$

Chi-squared $=21.03 ; d f=6 \quad(p=0.0018)$ Inconsistency $\left.\right|^{2}=71.5 \%$

Diagnostic OR
108.00
40.33
60.80
1275.00
39.80
8.50
28.13

Pooled diagnostic OR=35.83 (15.57-82.43) Cochran-Q=8.67; $d f=6(p=0.1932)$ Inconsistency $\left.\right|^{2}=30.8 \%$ Tau-squared $=0.3629$

FIGURE 6. Forest plots of sensitivity, specificity and diagnostic odds ratio of T-SPOT.TBв performed on extrasanguinous samples. Sensitivity data represent pooled values that were computed on all tuberculosis cases (culture-confirmed and non-confirmed cases). All studies reported data on both sensitivity and specificity. df: degrees of freedom.

The urgent need for alternative diagnostic assays is emphasised by the fact that $<50 \%$ of pulmonary TB cases, and therefore the majority of all TB suspects, have undetectable AFB in sputum smear examinations. While neither TST nor IGRAs are clinically relevant in AFB sputum smear-positive cases, rapid TB case detection must be improved in AFB sputum smear-negative patients, especially as $>10 \%$ of secondary TB cases are contracted from persons with negative AFB sputum smears [52]. In this situation, the T-SPOT.TB ${ }_{\circledR}$ assay, applied directly on extrasanguinous fluids, could be an important improvement for a rapid decision to initiate anti-TB treatment in countries of low TB incidence. In such low-incidence settings, such specimens are routinely collected in TB suspects with AFB sputum smearnegative TB and the ELISPOT technology is available [53].
The current meta-analysis has some limitations in that there was a considerable heterogeneity between studies. In addition, information on the performance of the assays in children is limited. Likewise, although many studies included immunocompromised patients, results are not reported in a stratified way that would allow the effect of immunosuppression to be addressed. Thus, more studies are needed that specifically assess the effect of immunodeficiency on diagnostic accuracy of the tests. This would be particularly relevant in the setting of drug-mediated immunosuppression and in regions with a high prevalence of HIV/M. tuberculosis co-infection. The calculation of specificities was based not only on TB suspects but also on individuals with a low risk for $M$. tuberculosis infection. While this heterogeneity may lead to an overestimation of specificity, 
this clearly emphasises the inability of IGRAs to distinguish active disease from latent infection as well as lack of infection.

In conclusion, the current evidence brought forward in this systematic review and meta-analysis shows that the IGRAs have limited accuracy in diagnosing active TB. It is essential to present and describe such evidence in guidance documents to ensure that the assays are only introduced and applied in diagnostic algorithms where they are of proven accuracy. Sensitivity of IGRAs performed on blood is superior to that of TST in patients with active TB. However, the low specificity may indicate limited value of IGRAs to distinguish between patients with specific immunity due to latent $M$. tuberculosis infection from patients with active disease. Conversely, as diagnostic accuracy is high when using T-SPOT.TB ${ }$ on extrasanguinous fluids, T-SPOT.TB $B_{\mathbb{R}}$ is currently the best available immunological method to distinguish active TB from latent M. tuberculosis infection. However, the number of patients analysed so far is low, evaluation relied on pooled data, and the application of IGRAs on extrasanguinous fluids for the diagnosis of active TB has not yet been evaluated by the regulatory authorities. Thus, definite evidence for the use of IGRAs from compartments other than blood will require more independent and carefully designed prospective studies.

\section{STATEMENT OF INTEREST}

Statements of interest for P. Ravn, A. Bossink and E. Girardi can be found at www.erj.ersjournals.com/site/misc/statements.xhtml

\section{ACKNOWLEDGEMENTS}

The affiliations of the present authors are as follows. M. Sester: Dept of Transplant and Infection Immunology, Saarland University, Homburg; C. Lange: on behalf of the TBNET, Clinical Infectious Diseases and Center for Clinical Studies, Medical Clinic, Research Center Borstel, Borstel; C. Giehl: European Research and Project Office GmbH - Eurice, Saarbrücken; R. Diel: Dept of Pulmonary Medicine, Hannover Medical School, Hannover (all Germany). G. Sotgiu: Hygiene and Preventive Medicine Institute, Sassari University, Sassari; E. Girardi: National Institute for Infectious Diseases L. Spallanzani, Rome; G.B. Migliori: WHO Collaborating Centre for TB and Lung Diseases, Fondazione S. Maugeri, Care and Research Institute, Tradate (all Italy). A. Bossink: Dept of Pulmonary Medicine, Diakonessenhuis, Utrecht, The Netherlands; K. Dheda: Lung Infection and Immunity Unit, Division of Pulmonology and University of Cape Town Lung Institute, Dept of Medicine, Cape Town, South Africa; J. Dominguez: Dept of Microbiology, Institut d'Investigació en Ciències de la Salut Germans Trias i Pujol, Ciber Enfermedades Respiratorias, Institutio de Salud Carlos III, Badalona, Spain; M. Lipman: Royal Free Hospital, London, UK; J. Nemeth: Universitätsspital Basel, Basel, Switzerland; P. Ravn: University Hospital Herlev, Herlev, Denmark; S. Winkler: Dept of Internal Medicine, Division of Infectious Diseases and Tropical Medicine, Medical University of Vienna, Vienna, Austria; E. Huitric, A. Sandgren and D. Manissero: European Centre for Disease Prevention and Control (ECDC), Stockholm, Sweden.

TBNET is a Clinical Research Collaboration (CRC) of the European Respiratory Society (ERS).

\section{REFERENCES}

1 Raviglione MC, Uplekar MW. WHO's new Stop TB Strategy. Lancet 2006; 367: 952-955.

2 European Centre for Disease Prevention and Control. Framework Action Plan to fight Tuberculosis in the European Union. Stockhom, 2008. Available from www.ecdc.europa.eu/en/publications/
Publications/0803_SPR_TB_Action_plan.pdf Date last accessed: July 7, 2010.

3 Pai M, O'Brien R. New diagnostics for latent and active tuberculosis: state of the art and future prospects. Semin Respir Crit Care Med 2008; 29: 560-568.

4 Newton SM, Brent AJ, Anderson S, et al. Paediatric tuberculosis. Lancet Infect Dis 2008; 8: 498-510.

5 WHO. Global tuberculosis control 2009. Epidemiology, strategy, financing. Geneva, WHO Report, 2009.

6 Mack U, Migliori GB, Sester M, et al. LTBI: latent tuberculosis infection or lasting immune responses to $M$. tuberculosis? A TBNET consensus statement. Eur Respir J 2009; 33: 956-973.

7 Young DB, Gideon HP, Wilkinson RJ. Eliminating latent tuberculosis. Trends Microbiol 2009; 17: 183-188.

8 Andersen P, Munk ME, Pollock JM, et al. Specific immune-based diagnosis of tuberculosis. Lancet 2000; 356: 1099-1104.

9 Cellestis. Quantiferon-TB $\mathbb{R}_{\mathbb{R}}$ Gold. 2009. www.cellestis.com/ Date last accessed: October 20, 2009. Date last updated: 2010.

10 OxfordImmunotec. T.Spot-TB®. 2009. www.oxfordimmunotec. com/ Date last accessed: October 20, 2009. Date last updated: 2010.

11 Barry CE 3rd, Boshoff HI, Dartois V, et al. The spectrum of latent tuberculosis: rethinking the biology and intervention strategies. Nat Rev Microbiol 2009; 7: 845-855.

12 Lange C, Pai M, Drobniewski F, et al. Interferon- $\gamma$ release assays for the diagnosis of active tuberculosis: sensible or silly? Eur Respir J 2009; 33: 1250-1253.

13 Dheda K, Schwander SK, Zhu B, et al. The immunology of tuberculosis: from bench to bedside. Respirology 2010; 15: 433-450.

14 Jafari C, Ernst M, Kalsdorf B, et al. Rapid diagnosis of smearnegative tuberculosis by bronchoalveolar lavage enzyme-linked immunospot. Am J Respir Crit Care Med 2006; 174: 1048-1054.

15 Jafari C, Ernst M, Strassburg A, et al. Local immunodiagnosis of pulmonary tuberculosis by enzyme-linked immunospot. Eur Respir J 2008; 31: 261-265.

16 Jafari C, Thijsen S, Sotgiu G, et al. Bronchoalveolar lavage enzymelinked immunospot for a rapid diagnosis of tuberculosis: a Tuberculosis Network European Trialsgroup study. Am J Respir Crit Care Med 2009; 180: 666-673.

17 Dheda K, van Zyl-Smit RN, Meldau R, et al. Quantitative lung T cell responses aid the rapid diagnosis of pulmonary tuberculosis. Thorax 2009; 64: 847-853.

18 Wilkinson KA, Wilkinson RJ, Pathan A, et al. Ex vivo characterization of early secretory antigenic target 6 -specific $\mathrm{T}$ cells at sites of active disease in pleural tuberculosis. Clin Infect Dis 2005; 40: 184-187.

19 Losi M, Bossink A, Codecasa L, et al. Use of a T-cell interferon- $\gamma$ release assay for the diagnosis of tuberculous pleurisy. Eur Respir $J$ 2007; 30: 1173-1179.

20 Thomas MM, Hinks TS, Raghuraman S, et al. Rapid diagnosis of Mycobacterium tuberculosis meningitis by enumeration of cerebrospinal fluid antigen-specific T-cells. Int J Tuberc Lung Dis 2008; 12: 651-657.

21 Moher D, Liberati A, Tetzlaff J, et al. Preferred reporting items for systematic reviews and meta-analyses: the PRISMA statement. PLoS Med 2009; 6: e1000097.

22 Whiting P, Rutjes AW, Reitsma JB, et al. The development of QUADAS: a tool for the quality assessment of studies of diagnostic accuracy included in systematic reviews. BMC Med Res Methodol 2003; 3: 25.

23 Whiting PF, Weswood ME, Rutjes AW, et al. Evaluation of QUADAS, a tool for the quality assessment of diagnostic accuracy studies. BMC Med Res Methodol 2006; 6: 9.

24 Zamora J, Abraira V, Muriel A, et al. Meta-DiSc: a software for metaanalysis of test accuracy data. BMC Med Res Methodol 2006; 6: 31.

25 Baba K, Sornes S, Hoosen AA, et al. Evaluation of immune responses in HIV infected patients with pleural tuberculosis by the QuantiFERON TB-Gold interferon- $\gamma$ assay. BMC Infect Dis 2008; 8: 35. 
26 Bamford AR, Crook AM, Clark JE, et al. Comparison of interferon- $\gamma$ release assays and tuberculin skin test in predicting active tuberculosis (TB) in children in the UK: a paediatric TB network study. Arch Dis Child 2010; 95: 180-186.

27 Bartu V, Havelkova M, Kopecka E. QuantiFERON-TB Gold in the diagnosis of active tuberculosis. J Int Med Res 2008; 36: 434-437.

28 Bianchi L, Galli L, Moriondo M, et al. Interferon- $\gamma$ release assay improves the diagnosis of tuberculosis in children. Pediatr Infect Dis J 2009; 28: 510-514.

29 Bosshard V, Roux-Lombard P, Perneger T, et al. Do results of the T-SPOT.TB interferon- $\gamma$ release assay change after treatment of tuberculosis? Respir Med 2009; 103: 30-34.

30 Chee CB, Gan SH, Khinmar KW, et al. Comparison of sensitivities of two commercial $\gamma$ interferon release assays for pulmonary tuberculosis. J Clin Microbiol 2008; 46: 1935-1940.

31 Chegou NN, Walzl G, Bolliger CT, et al. Evaluation of adapted whole-blood interferon- $\gamma$ release assays for the diagnosis of pleural tuberculosis. Respiration 2008; 76: 131-138.

32 Dheda K, van Zyl-Smit RN, Sechi LA, et al. Utility of quantitative T-cell responses versus unstimulated interferon- $\gamma$ for the diagnosis of pleural tuberculosis. Eur Respir J 2009; 34: 1118-1126.

33 Dominguez J, De Souza-Galvao M, Ruiz-Manzano J, et al. T-cell responses to the Mycobacterium tuberculosis-specific antigens in active tuberculosis patients at the beginning, during, and after antituberculosis treatment. Diagn Microbiol Infect Dis 2009; 63: 43-51.

34 Eum SY, Lee YJ, Kwak HK, et al. Evaluation of the diagnostic utility of a whole-blood interferon- $\gamma$ assay for determining the risk of exposure to Mycobacterium tuberculosis in Bacille CalmetteGuerin (BCG)-vaccinated individuals. Diagn Microbiol Infect Dis 2008; 61: 181-186.

35 Goletti D, Carrara S, Butera O, et al. Accuracy of immunodiagnostic tests for active tuberculosis using single and combined results: a multicenter TBNET-Study. PLOS ONE 2008; 3: e3417.

36 Goletti D, Carrara S, Vincenti D, et al. Accuracy of an immune diagnostic assay based on RD1 selected epitopes for active tuberculosis in a clinical setting: a pilot study. Clin Microbiol Infect 2006; 12: 544-550.

37 Janssens JP, Roux-Lombard P, Perneger T, et al. Quantitative scoring of an interferon- $\gamma$ assay for differentiating active from latent tuberculosis. Eur Respir J 2007; 30: 722-728.

38 Kampmann B, Whittaker E, Williams A, et al. Interferon- $\gamma$ release assays do not identify more children with active tuberculosis than the tuberculin skin test. Eur Respir J 2009; 33: 1374-1382.
39 Kim SH, Cho OH, Park SJ, et al. Diagnosis of abdominal tuberculosis by T-cell-based assays on peripheral blood and peritoneal fluid mononuclear cells. J Infect 2009; 59: 409-415.

40 Kim SH, Choi SJ, Kim HB, et al. Diagnostic usefulness of a T-cell based assay for extrapulmonary tuberculosis. Arch Intern Med 2007; 167: 2255-2259.

41 Kim SH, Song KH, Choi SJ, et al. Diagnostic usefulness of a T-cellbased assay for extrapulmonary tuberculosis in immunocompromised patients. Am J Med 2009; 122: 189-195.

42 Kobashi Y, Mouri K, Yagi S, et al. Clinical evaluation for diagnosing active $\mathrm{TB}$ disease and transitional change of two commercial blood tests. Scand J Infect Dis 2008; 40: 629-634.

43 Lee LN, Chou CH, Wang JY, et al. Enzyme-linked immunospot assay for interferon- $\gamma$ in the diagnosis of tuberculous pleurisy. Clin Microbiol Infect 2009; 15: 173-179.

44 Meier T, Eulenbruch HP, Wrighton-Smith P, et al. Sensitivity of a new commercial enzyme-linked immunospot assay (T-SPOT.TB) for diagnosis of tuberculosis in clinical practice. Eur I Clin Microbiol Infect Dis 2005; 24: 529-536.

45 Nicol MP, Davies MA, Wood K, et al. Comparison of T-SPOT.TB assay and tuberculin skin test for the evaluation of young children at high risk for tuberculosis in a community setting. Pediatrics 2009; 123: 38-43.

46 Syed Ahamed Kabeer B, Sikhamani R, Swaminathan S, et al. Role of interferon- $\gamma$ release assay in active TB diagnosis among HIV infected individuals. PLoS One 2009; 4: e5718.

47 Tsiouris SJ, Coetzee D, Toro PL, et al. Sensitivity analysis and potential uses of a novel $\gamma$ interferon release assay for diagnosis of tuberculosis. J Clin Microbiol 2006; 44: 2844-2850.

48 Diel R, Loddenkemper R, Nienhaus A. Evidence-based comparison of commercial interferon- $\gamma$ release assays for detecting active TB: a metaanalysis. Chest 2010; 137: 952-968.

49 Mori T. Usefulness of interferon- $\gamma$ release assays for diagnosing TB infection and problems with these assays. J Infect Chemother 2009, 15: 143-155.

50 Richeldi L. An update on the diagnosis of tuberculosis infection. Am J Respir Crit Care Med 2006; 174: 736-742.

51 Dosanjh DP, Hinks TS, Innes JA, et al. Improved diagnostic evaluation of suspected tuberculosis. Ann Intern Med 2008; 148 325-336.

52 Tostmann A, Kik SV, Kalisvaart NA, et al. Tuberculosis transmission by patients with smear-negative pulmonary tuberculosis in a large cohort in the Netherlands. Clin Infect Dis 2008; 47: 1135-1142.

53 Lange C, Mori T. Advances in the diagnosis of tuberculosis Respirology 2010; 15: 220-240. 